\title{
Journal of Counseling Psychology Principal Reviewers, Contributing Reviewers and Pre-Doctoral Co-Reviewers (September 2015 to September 2016)
}

\section{PRINCIPAL REVIEWERS}

The Editor and Associate Editors wish to thank the following persons who served as principal reviewers for Journal of Counseling Psychology by reviewing a minimum of four manuscripts.

Melanie Elyse Brewster

Stephanie L. Budge

Ruth Chu-Lien Chao

Noah M. Collins

Patton O. Garriott
Joshua N. Hook

Sharon G. Horne

Carla D. Hunter

Dennis (Martin) Kivlighan

Ryon McDermott
Takuya Minami

Jae Puckett

Mike Schaub

Jessica V. Stahl

\section{CONTRIBUTING REVIEWERS}

Stefanía Ægisdóttir

Blake A. Allan

Mary Z. Anderson

Dana Atzil-Slonim

Eran Bar-Kalifa

Bianca L. Bernstein

Kathleen J. Bieschke

Lynette H. Bikos

Sha'Kema Blackmon

Gary Burlingame

Grace I. L. Caskie

Edward C. Chang

Tai Chang

Norah Chapman

Rachel Gali Cinamon

Kevin Cokley

Collie W. Conoley

Patrick Corrigan

Lisa De La Rue

Edward A. Delgado-Romero

David M. Dunkley

Sif Einarsdóttir

Robert Elliott

Carol Falender

Fredrik Falkenström

Ann R. Fischer

Bryana French

Myrna Friedlander

Kristen Adams

Bonnie Andrews

Annabelle Atkin

Brittany Carbaugh

Selda Celen

Samantha Cruz

Nicholas Davey
Omar Carlo Gioacchino Gelo

Cynthia E. Glidden-Tracey

Rodney K. Goodyear

Joseph H. Hammer

Marie S. Hammond

Silva Hassert

Laurie Heatherington

Nick Heck

Jennifer Henretty

Keith C. Herman

Michiyo Hirai

Andreas Hirschi

Christopher Hopwood

Adam O. Horvath

Zhi-Jin Hou

Bong Joo Hwang

Arpana G. Inman

Margo A. Jackson

Chad V. Johnson

LaRae M. Jome

Ae Kyung Jung

Merle Keitel

Harrison J. Kell

Maureen E. Kenny

Sung Kim

Michael Y. Lau

Richard M. Lee

Jessica Nina Lester
Ronald F. Levant

Michael E. Levin

Jioni A. Lewis

Chris Liang

Hsin-Ya Liao

James W. Lichtenberg

Marcia Mei-Lee Liu

William Ming Liu

Gianluca Lo Coco

Michael I. Loewy

P. Priscilla Lui

Heather Z. Lyons

Patrick M. Markey

Suzanne E. Mazzeo

John McConnell

John McLeod

Ethan H. Mereish

Uma Chandrika Millner

Debra Mollen

Nancy L. Murdock

Rachel L. Navarro

Dana Nelson

Jill Denise Paquin

Sampo Paunonen

Bridgette Peteet

Gary Peterson

Zoe Peterson

Robert J. Reese
Brandon L. Velez

David L. Vogel

\section{CONTRIBUTING PRE-DOCTORAL CO-REVIEWERS}

Brittan Davis

Tohar Dolev

Melissa Ertl

Jack Haeger

Patrick Heath

Aylin Kaya
Dean Malec

Meredith Maroney

Eric R. McCurdy

Michael Murgo

Brian Pace

Ashley Essel Poklar

Yael Porat
Christine Robitschek Patrick J. Rottinghaus James Rounds

Dominik Schoebi

Christopher Slaten

Paul M. Spengler

Graham Stead

Lara M. Stepleman

Joachim Stoeber

Linda Mezydlo Subich

Martin Swanbrow Becker

Giorgio A. Tasca

Elliot A. Tebbe

Marilyn Thompson

David M. Tokar

Michael Waldo

Chiachih DC Wang

Serena Wee

Meifen Wei

Susan C. Whiston

Bart Wille

Lydia Woodyatt

Aidan Wright

Gerhard Zarbock

Heidi Zetzer

Sigal Zilcha-Mano
Tangela Roberts

Chen Roei

Radinka J. Samardzic

Courtney Simpson

Nancy N. Truong

Erica Wiley Whiteman 